\title{
Revisiting the potential advantage of robotic surgical system in spleen-preserving distal pancreatectomy over conventional laparoscopic approach
}

\author{
Seok Jeong Yang ${ }^{1}$, Ho Kyoung Hwang ${ }^{2,3}$, Chang Moo Kang ${ }^{2,3}$, Woo Jung Lee ${ }^{1}$ \\ ${ }^{1}$ Department of Surgery, Yonsei University College of Medicine, Yongin Severance Hospital, Gyeonggi, Korea; ${ }^{2}$ Division of Hepatobiliary and \\ Pancreatic Surgery, Yonsei University College of Medicine, ${ }^{3}$ Pancreatobiliary Cancer Center, Yonsei Cancer Center, Severance Hospital, Seoul, \\ Korea \\ Contributions: (I) Conception and design: CM Kang; (II) Administrative support: WJ Lee; (III) Provision of study materials or patients: CM Kang, \\ HK Hwang; (IV) Collection and assembly of data: CM Kang, SJ Yang; (V) Data analysis and interpretation: SJ Yang; (VI) Manuscript writing: All \\ authors; (VII) Final approval of manuscript: All authors. \\ Correspondence to: Chang Moo Kang, MD, PhD. Department of Surgery, Yonsei University College of Medicine, Severance Hospital 50 Yonsei-Ro, \\ Seodaemun-Ku, Seoul, Korea. Email: cmkang@yuhs.ac.
}

Background: This study aimed to compare success rate of spleen preservation between robotic and laparoscopic distal pancreatectomy (DP).

Methods: Between November 2007 and March 2018, forty-one patients underwent the conventional laparoscopic DP (Lap group) and the other 37 patients underwent robotic DP (Robot group). The perioperative clinicopathologic variables were compared.

Results: The robotic procedure was chosen by younger patients compared to conventional laparoscopic surgery $(42.9 \pm 14.0$ vs. $51.3 \pm 14.6$ years, $\mathrm{P}=0.016)$. The mean operation time was longer $(313$ vs. $246 \mathrm{~min}$, $\mathrm{P}=0.000)$, but the mean tumor size was smaller in Robot group $(2.7 \pm 1.2$ vs. $4.2 \pm 3.3 \mathrm{~cm}, \mathrm{P}=0.018)$. The overall spleen-preserving rate was higher in the Robot group $(91.9 \%$ vs. $68.3 \%, \mathrm{P}=0.012)$. However, with accumulating laparoscopic experiences (after 16th case), the statistical differences in spleen preservation rate between the Robot and Lap groups had diminished ( $\mathrm{P}=0.428)$.

Conclusions: The present results suggest a robot can be helpful to save the spleen during DP for benign and borderline malignancy. However, a surgeon highly experienced in the laparoscopic approach can also produce a high success rate of spleen preservation, similar to that shown with the robotic approach.

Keywords: Robot; laparoscopic; spleen preservation; distal pancreatectomy

Submitted Nov 14, 2019. Accepted for publication Jan 10, 2020.

doi: $10.21037 /$ atm.2020.01.80

View this article at: http://dx.doi.org/10.21037/atm.2020.01.80

\section{Introduction}

With advances in axial image technology, there is an increasing number of patients being diagnosed with benign or borderline (low-grade) malignant tumors of the pancreas (1-3). They are expected to have excellent long-term survival rates, and their quality of life should be considered when choosing a surgical option. Therefore, functionpreserving and minimally invasive pancreatectomy is ideal (4-6), Surgical intervention is especially applicable when a low-grade malignant tumor is located in the pancreatic body or tail, and it is reasonable to attempt spleen-preserving minimally invasive distal pancreatectomy first (7-10); however, the procedure requires finely honed skills and advanced surgical techniques. Sometimes, surgeons abandon spleen salvation because of the technical complexity of dissecting splenic vessels with conventional laparoscopy (11). Alternatively, Warshaw et al. (12) proposed the technical feasibility of a splenic-vessel-sacrificing spleen-preserving 
distal pancreatectomy for simplifying surgical procedures, but there is the potential risk of left-sided portal hypertension-related perigastric varices and severe splenic infarction $(13,14)$.

Recently, robotic surgical systems have been applied to various kind of surgery. When it comes to distal pancreatectomy, surgeons expect robots to be able to help preserve the spleen. After Waters et al. (15) reported the cost effectiveness and higher spleen preservation rate of robotic procedures, investigations have been published supporting the idea that robot surgery is superior to conventional laparoscopy for spleen preservation during distal pancreatectomy $(2,15,16)$. In addition, several recent meta-analyses have demonstrated the high rate of spleen preservation in robotic distal pancreatectomies compared with the laparoscopic approach $(17,18)$. Previously, we also reported how the three-dimensional vision and endowrist motions of robots can promote spleen salvation during distal pancreatectomy, suggesting there is a clinical advantage to the robotic surgical system (19).

However, there are still conflicting data on the superiority of the spleen-preserving capacity of the robotic distal pancreatectomy (20-23). In addition, with surgeons' accumulating experience of the laparoscopic approach for distal pancreatectomy and the safety of alternative surgical techniques for splenic-vessel sacrificing spleenpreserving distal pancreatectomy $(13,24-26)$, we found that a satisfactory spleen preservation rate could be achieved even with conventional laparoscopy in cases when spleenpreserving distal pancreatectomy is preoperatively planned.

In this study, we reanalyzed the spleen-preserving capacity of robot-assisted and conventional laparoscopic distal pancreatectomy techniques for benign and borderline malignant tumors of the pancreas to investigate which approach would be most beneficial for spleen preservation during minimally invasive distal pancreatectomy.

\section{Methods}

This retrospective study protocol was approved by the Yonsei University Institutional Review Board (IRB No. 42015-1124). We reviewed the medical records of patients who underwent minimally invasive distal pancreatectomy for benign and borderline malignant tumors, which were performed by a single surgeon from November 2007 to March 2018. The surgeon began robotic distal pancreatectomy in September 2007 after experiencing nine cases of conventional laparoscopic distal pancreatectomy since July 2006. For benign and low malignant tumors of the distal pancreas, our principle of surgery is spleenpreserving distal pancreatectomy (SpDP), unless combined splenectomy is required for medical reasons. For spleen preservation, initial surgical strategy is always splenic vessel conservation. In brief, for small tributary vessels between splenic vessels and pancreas can be carefully dissected by articulating two robotic arms. with left-sided pancreas retracted by using third robotic $3^{\text {rd }}$ arm. These vessels can be controlled by either suture ligation, small metal clips, and energy device. Accurate robotic movement without tremor is thought to be great advantage for this surgical procedure. Especially, pancreatic tail is located behind the splenic hilum, similarly, tip of the pancreatic tail can be effectively dissected off from the splenic hilum $(16,27)$. Only if vessel dissection is too difficult or hazardous, splenic-vessel-sacrificing SVS-SpDP is performed. Concurrent splenectomy is always the last option. When dividing the pancreas, endo-GIA was applied following the surgical concept of prolonged peri-firing sequential compression stapling technique (28). When pancreatic duct was identified on the staple-line, some small metal clips or suture ligation was added by using robotic instruments.

Surgical indications for minimally invasive distal pancreatectomy were the same for conventional laparoscopy or robotic surgery, as described in previous report $(16,19,27)$. Patient decided on their surgical approach (laparoscopy or robot) after being fully informed about its characteristics, operation results, and cost. The robotic surgical systems were the daVinci S or Si surgical system (Intuitive Surgical, Sunnyvale, CA).

The perioperative variables, such as age, sex, body mass index, operation time, bleedings, spleen conservation, etc., were compared between the two groups. Postoperative complications were recorded according to Clavien-Dindo classification. If the complication was more than class III, it was defined as a morbid complication. Postoperative pancreas fistulas (POPF) were graded according to the suggestion by international study group of pancreas fistula (ISGPF) and a clinically relevant POPF (CR-POPF) was mentioned when the fistula was more than grade $\mathrm{B}$, according to ISGPF (29). To compare the efficacy of spleen preservation between the two modalities, the cumulative spleen-preserving rate (CSPR), which was introduced in our previous report as an intention-to-treat conception, was investigated before and after the learning curve (17).

Continuous variables were expressed as mean \pm standard deviation or range and categorical variables as frequency 


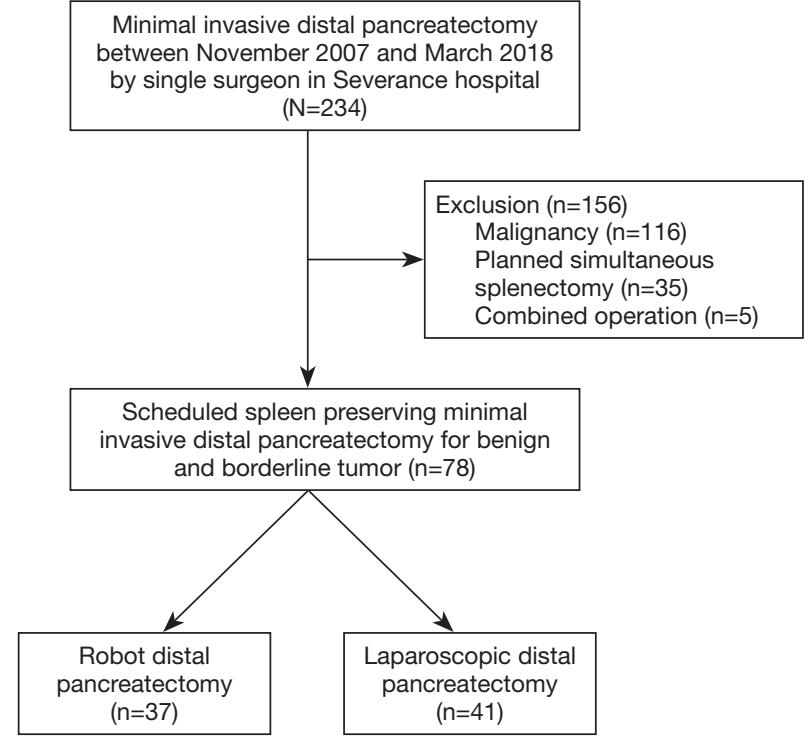

Figure 1 Flow diagram showing patient enrollment for scheduled spleen-preserving minimally invasive distal pancreatectomy.

(\%). Student's-t-test and chi-square test or Fisher's exact test were used to analyze continuous and categorical variables. Statistical analyses were performed using SPSS 20.0 for Windows (SPSS Inc., Chicago, IL, USA). Data were considered statistically significant when $\mathrm{P}$ values $<0.05$.

\section{Results}

\section{Short-term perioperative surgical outcomes between robotic and laparoscopic distal pancreatectomy}

A total of 234 patients underwent minimally invasive distal pancreatectomy, performed by a single surgeon in Severance Hospital. Among them, 116 cases for malignancy, 35 cases for initial planned simultaneous splenectomy, and 5 cases for combined operation were excluded (Figure 1). Seventy-eight patients ( 37 cases by robot and 41 cases by laparoscopy) were enrolled in this study, and intended spleen-preserving minimally invasive distal pancreatectomy was attempted first for left-sided pancreas benign and borderline malignant tumors (Table 1). The Robot group included younger patients than the laparoscopy group $(42.9 \pm 14.0$ vs. $51.3 \pm 14.6$ years, $\mathrm{P}=0.016)$ and smaller tumors $(2.7 \pm 1.2$ vs. $4.2 \pm 3.3 \mathrm{~cm}, \mathrm{P}=0.018)$. The resected pancreas length seemed to be shorter in the Robot group $(7.6 \pm 3.3$ vs. $9.4 \pm 4.2 \mathrm{~cm}, \mathrm{P}=0.051)$, but the difference did not achieve statistical significance. The mean operation time was longer in the Robot group (313 vs. $246 \mathrm{~min}, \mathrm{P}=0.000$ ). There were no statistical differences for other perioperative outcomes, such as bleeding amount, transfusions, overall morbidity, clinically relevant postoperative pancreatic fistula, or postoperative hospital stay, between the two groups.

\section{Experience-dependent changes in the cumulative spleen- preserving rate between robot and laparoscopic distal pancreatectomy}

The overall spleen preservation rate was higher in the Robot group (91.9\% vs. 68.3\%, $\mathrm{P}=0.012$ ). Among overall spleen preservation cases, the Robot group yielded a higher spleen vessel conservation rate than laparoscopy $(73.0 \% \mathrm{vs}$. $39.0 \% ; \mathrm{P}=0.006)$. In addition, the overall CSPR was higher in the Robot group. However, with the surgeon's increasing experience of laparoscopic distal pancreatectomies, the difference in the CSPR between robot and conventional laparoscopy became narrower (Figure 2). After 16 consecutive cases by conventional laparoscopy, the statistical differences, in terms of CSPR $(94.7 \%$ vs. $80.0 \%, \mathrm{P}=0.428)$ and splenic vessel conservation rate $(66.7 \%$ vs. $32.0 \%$, $\mathrm{P}=0.068$ ), between the robot and conventional laparoscopic groups disappeared (Table 2, Figure 3).

\section{Discussion}

Despite the global applications of minimal invasive technique in all fields of abdominal operation, laparoscopic pancreas surgery has not been widely accepted for a while, due to the complexity of anatomy and the risk of complications $(30,31)$. However, for left-sided pancreatectomy, many studies have been reported the safety and even superiority of laparoscopic approach over open method, making the laparoscopic distal pancreatectomy as a standard surgery (32). With improvement of endostapler devices and the prolonged peri-firing sequential compression stapling technique to minimize the break of pancreas stump, a significant reduction of the CR$\mathrm{POPF}$ is one of the reasons why laparoscopic approach is well accepted in the distal pancreatectomy than pancreaticoduodenectomy (28). Song et al. reported $7 \%$ of CR-POPF in their article about 359 consecutive laparoscopic DP without any postoperative mortality and Røsok et al. showed 6-8\% of ISGPF B/C POPF after open and minimal invasive distal pancreatectomy in their systemic review $(32,33)$. In this study, there were five patients $(6.4 \%)$ with CR-POPF, but all of them were ISGPF grade B. 
Table 1 Demographic and clinical comparative analysis between laparoscopic and robotic distal pancreatectomy groups

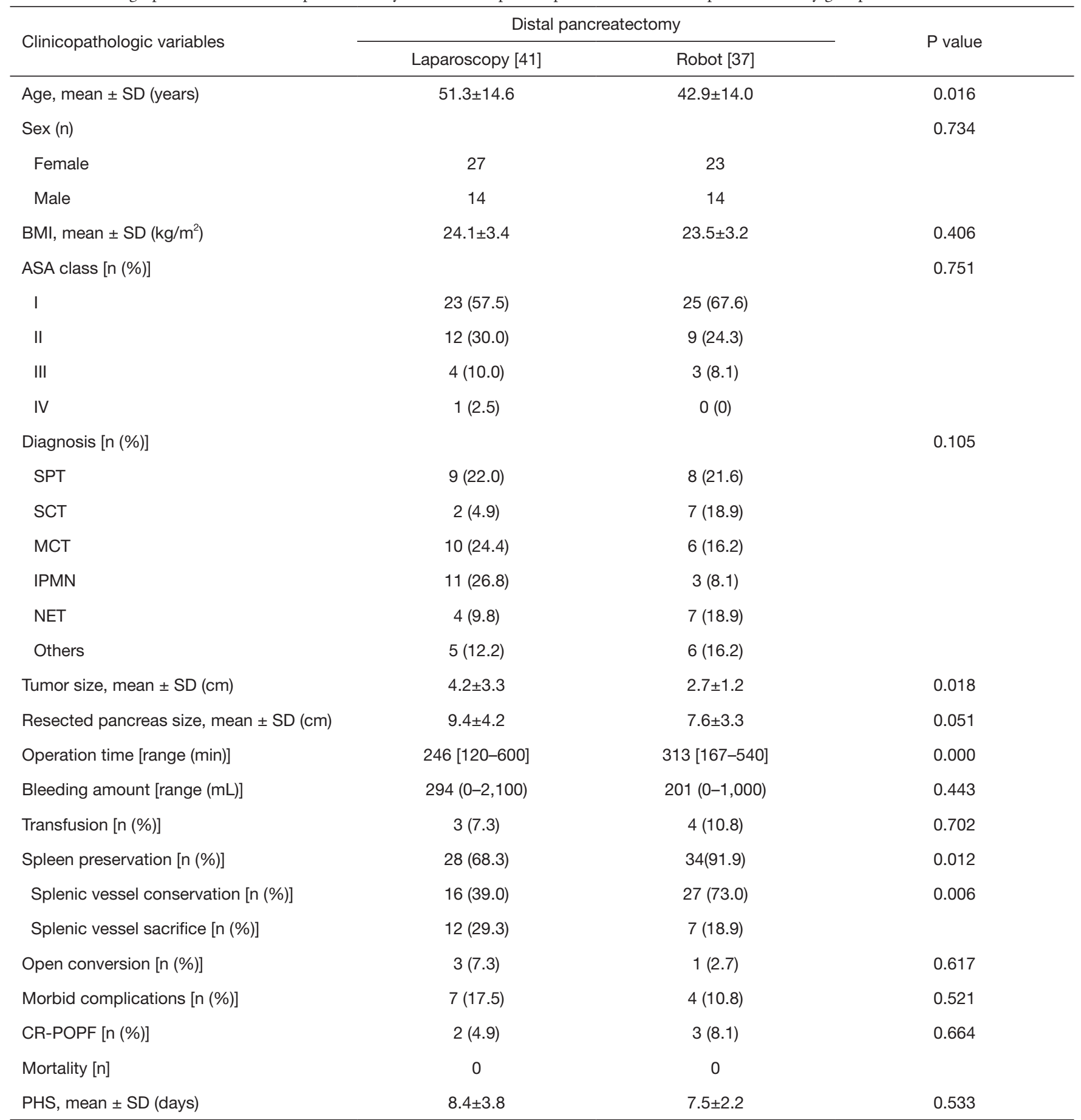

$\mathrm{SD}$, standard deviation; BMI, body mass index; ASA, American society of anesthesiologists physical status classification system; SPT, solid pseudopapillary tumor; SCT, serous cystic tumor; MCT, mucinous cystic tumor; IPMN, intraductal papillary mucinous neoplasm; NET, neuroendocrine tumor; CR-POPF, clinically relevant postoperative pancreas fistula; PHS, postoperative hospital stay; Statistical significance at $\mathrm{P}<0.05$. 
Based on these reports on the safety of laparoscopic distal pancreatectomy, many surgeons attempted more complex laparoscopic operation for the lesion in the pancreas body and tail, like as pancreas parenchyma preservation, spleen preservation or reduced port surgery, but they often encountered technical problems during these advanced surgeries with conventional laparoscopy (34).

Following the invention of the robotic surgical system, many surgeons have been fascinated by its threedimensional vision, wide range of endo-wrist movements, and computer-based platform. In particular, in the field of pancreatic surgery, surgeons expected the robot surgical system to help them accomplish complex distal pancreas surgery, such as spleen preserving distal pancreatectomy for low-grade malignant tumors $(2,16,19,35)$. In fact, we previously demonstrated the potential clinical benefit of the robotic surgical system for spleen preservation when performing distal pancreatectomy. Although robotic

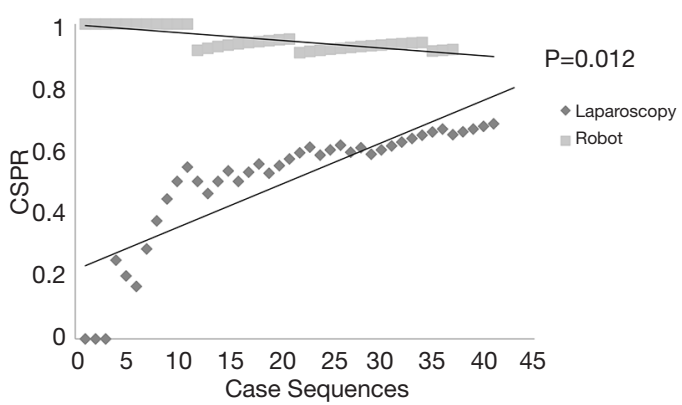

Figure 2 Overall cumulative spleen-preserving rate (CSPR) was higher for robot than conventional laparoscopy. Statistical significance at $\mathrm{P}<0.05$. surgery is expensive, robotic distal pancreatectomy has the advantage of a high success rate of spleen preservation (9/16 vs. $1 / 19, \mathrm{P}=0.027)(19)$. In addition, many other studies have demonstrated similar favorable outcomes in terms of the spleen preservation rate in robotic distal pancreatectomy $(2,15,36,37)$.

It is well known that splenectomy increases not only the risk of postoperative infectious complications but also the life-long probability of hospitalization or death from sepsis or cancer (38-41). Therefore, if there is no medical need for concurrent splenectomy, spleen preservation has been recommended during distal pancreatectomy for benign and borderline malignant tumors in distal pancreas $(8,42)$.

There are two options for SpDP. One is splenic-vesselconserving SpDP (SVC-SpDP), the other is splenic-vesselsacrificing SpDP (SVS-SpDP), the so-called Warshaw operation $(43,44)$. Even though SVS-SpDP is a good alternative when splenic-vessel saving is difficult, it can cause splenic infarction, perigastric varices, and chronic left abdominal pain. Therefore, SVC-SpDP seems to be the more attractive technique $(45,46)$. However, splenic vessels have many small and fragile branches within the pancreas, and often the pancreatic tissue itself encases splenic vessels. These anatomic characteristics make spleen preservation too problematic when performing conventional laparoscopic distal pancreatectomy. In contrast, the stable $3 \mathrm{D}$-operating vision, fine dissecting and clipping system, and the multidirectional endo-wrist function of a robotic surgical system is thought to be very suitable for $\mathrm{SpDP}$, as already demonstrated in our previous reports $(16,19)$. In particular, the spleen hilum has many vessels intertwined with the pancreas, so inappropriate access or even a

Table 2 Spleen preservation rate of conventional laparoscopy and robot groups before and after learning curve ( $\mathrm{n}=16$ ) for laparoscopic distal pancreatectomy

\begin{tabular}{|c|c|c|c|}
\hline & \multicolumn{2}{|c|}{ Distal pancreatectomy } & $P$ value \\
\hline \multicolumn{4}{|l|}{ Before learning curve } \\
\hline Spleen preservation [n/N (\%)] & $8 / 16(50.0)$ & $16 / 16(93.8)$ & 0.015 \\
\hline Splenic vessel conservation [n/N (\%)] & $8 / 16(50.0)$ & $13 / 16(81.3)$ & 0.009 \\
\hline Spleen preservation [n/N (\%)] & $20 / 25(80.0)$ & 19/21 (94.7) & 0.428 \\
\hline Splenic vessel conservation [n/N (\%)] & $8 / 25(32.0)$ & $14 / 21(66.7)$ & 0.068 \\
\hline
\end{tabular}

Statistical significance at $\mathrm{P}<0.05$. 


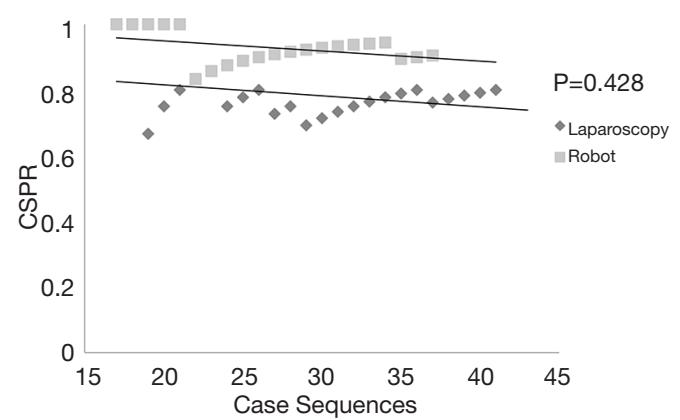

Figure 3 Statistical difference disappeared for cumulative spleen-preserving rate (CSPR) after $16^{\text {th }}$ laparoscopy. Statistical significance at $\mathrm{P}<0.05$.

small unnecessary movement in the use of laparoscopic instrument can cause large amounts of bleeding from these branches to abandon spleen preservation, but the robot can overcome this obstacle by effective 3 rd arm movement and angulating motion of the robotic arms without tremor $(27,36)$.

While increasing our experience of both laparoscopic and robotic distal pancreatectomies, we have encountered some cases for which the conventional laparoscopic approach might be more convenient than the robotic distal pancreatectomy. There are potential techniques of SVSSpDP that can increase the success rate of laparoscopic spleen-preserving distal pancreatectomy $(11,16,44)$. Pancreatic cystic tumors or small vessels around the pancreas may be lacerated during the robotic surgical procedure, because there is no tactile feedback in this system. In addition, the conventional laparoscopy does not require additional time for the docking process, and laparoscopic working instruments can move quickly without any time lag, as occurs with the robotic system. Moreover, robotic surgery has been blamed for its higher cost without obviously superior results compared to laparoscopy. Therefore, based on our increasing experience of the laparoscopic approach, we tried to revisit the efficacy of the robotic surgical system for spleen-preserving distal pancreatectomy $(23,47,48)$.

In this study, the robot seems to be beneficial for overall successful SpDP (91.9\% vs. 68.3\%, $\mathrm{P}=0.012)$ with splenic vessel conservation $(73.0 \%$ vs. $39.0 \% ; \mathrm{P}=0.006)$. However, as the number of cases increased, the difference in CSPR between robot and laparoscopy became narrower (Figure 2). This can be interpreted as the benefit of the robotic system being attenuated by accumulating laparoscopy experiences.
Yang et al. Spleen preservation rate between robot and laparoscopy

At last, the statistical difference in the spleen preservation rate between the two approaches disappeared $(94.7 \% v$ s. $80.0 \%, \mathrm{P}=0.428$, Figure 3, Table 2) after the accumulation of experience of laparoscopic distal pancreatectomies (over 16 cases). This phenomenon also appeared to affect the splenic vessel conservation rate ( $66.7 \%$ vs. $32.0 \%, \mathrm{P}=0.068$, Table 2$)$. These results suggest that the robot has technical merits with regards to the meticulous vessel dissection during the spleen-preserving procedure, but the laparoscopic approach can also be used to achieve the surgical goal of minimally invasive spleen salvation if the surgeon has sufficient experience.

Consecutive experiences of laparoscopic distal pancreatectomy will develop surgical techniques and influence the selection of the most appropriate procedure. Hua et al. (49) also reported that surgeons' experiences of more than 15 laparoscopic distal pancreatectomies was associated with a reduced risk of conversion, and Kim et al. (50) suggested that 16 cases should be the cutoff point for safe splenic-vessel-preserving laparoscopic distal pancreatectomy. Similarly, Nachmany et al. (51) suggested that the surgical experience of at least 17 patients was needed for stabilizing perioperative surgical outcomes. To the contrary, for robot, Shyr et al. and Shakir reported 37 or 40 cases of robotic distal pancreatectomy for learning curve in their 70 and 100 cases series (52,53). Most learning curve analysis is based on operation time, and robotic surgery basically prolongs operation time. However, from the view point of spleen-preserving, robot can achieve higher spleen preservation rates (91.9\%) than laparoscopy $(68.3 \%)$ in spite of longer operation time in. In our study, we found that the CSPR seemed to increase after approximately 16 cases of laparoscopic distal pancreatectomy, but, robot yielded high spleen preservation rate from the beginning $(19,35)$.

The absence of tactile sense, which is said to be an important drawback of robotic surgery, can be overcome by visual sense through magnified 3D optical signal in robotic surgical system. In addition, additional docking time and time for changing frequent surgical instrument are concerned as disadvantage of robotic surgical system, which can be all resolved with accumulating experiences of robotic surgical team. However, emergent compensation toward unexpected event, such as massive bleeding, during robotic surgery still can be debated. Therefore, careful dissection should be kept in mind and laparoscopic resection followed by robotic reconstruction can be good alternative approach to overcome this issue $(54,55)$.

Therefore, in order to evaluate the potential role of the 
spleen-preserving power in both laparoscopic and robotic surgical approaches, the surgeons' experiences in different types of surgical approaches need to be considered. From the view-point of spleen preservation, for surgeons with little experiences of LDP, the robotic approach may be beneficial; however, with increasing experiences of LDP, both surgical approaches, such as laparoscopic and robotic, will be able to achieve the goal of spleen preservation by minimally invasive surgery. The most appropriate surgical approach should be carefully chosen according to the patient's condition, disease characteristics, and the surgeon's experience, as robotic surgery is still expensive, and not every patient can afford such high-cost surgery.

This study has some unavoidable limitations as a retrospective study. The first hurdle in comparing two surgical modalities is selection bias. Because the impact of surgery is critical, the surgeon has a tendency not to apply expensive new techniques for complicated cases, so we thought that was why smaller average tumor size in robot group than laparoscopy group. On the other hand, this selection bias may suggest that either robotic, or conventional laparoscopic approach should be carefully applied for successful spleen preserving procedure, considering the size of the tumor. Second, we only evaluated obvious perioperative outcomes and the spleen preservation rate, as they could be obtained in routine medical records. Robotic surgery has unique advantages that cannot be measured, such as the surgeon's ergonomic benefit, integrated image systems on the operating monitor, an effective tutorial system with double console, and a reduction in the need for assistance in laparoscope holding.

In conclusion, advanced robotic medical technology can help improve the success rate of spleen and splenic vessel preservation during distal pancreatectomy. However, conventional laparoscopy also can achieve safe and effective SpDP based on accumulating laparoscopic experiences. It is likely that, in the near future, surgeons will apply techniques from both robotic and laparoscopic approaches to perform safe and effective minimally invasive pancreatectomy.

\section{Acknowledgments}

Funding: None.

\section{Footnote}

Conflicts of Interest: The authors have no conflicts of interest to declare.
Ethical Statement: The authors are accountable for all aspects of the work in ensuring that questions related to the accuracy or integrity of any part of the work are appropriately investigated and resolved. This study was approved by the Yonsei University Institutional Review Board (IRB No. 42015-1124).

Open Access Statement: This is an Open Access article distributed in accordance with the Creative Commons Attribution-NonCommercial-NoDerivs 4.0 International License (CC BY-NC-ND 4.0), which permits the noncommercial replication and distribution of the article with the strict proviso that no changes or edits are made and the original work is properly cited (including links to both the formal publication through the relevant DOI and the license). See: https://creativecommons.org/licenses/by-nc-nd/4.0/.

\section{References}

1. Laffan TA, Horton KM, Klein AP, et al. Prevalence of unsuspected pancreatic cysts on MDCT. AJR Am J Roentgenol 2008;191:802-7.

2. Chen S, Zhan Q, Chen JZ, et al. Robotic approach improves spleen-preserving rate and shortens postoperative hospital stay of laparoscopic distal pancreatectomy: a matched cohort study. Surg Endosc 2015;29:3507-18.

3. de Jong K, Nio CY, Hermans JJ, et al. High prevalence of pancreatic cysts detected by screening magnetic resonance imaging examinations. Clin Gastroenterol Hepatol 2010;8:806-11.

4. Nakamura M, Nakashima H. Laparoscopic distal pancreatectomy and pancreatoduodenectomy: is it worthwhile? A meta-analysis of laparoscopic pancreatectomy. J Hepatobiliary Pancreat Sci 2013;20:421-8.

5. Jusoh AC, Ammori BJ. Laparoscopic versus open distal pancreatectomy: a systematic review of comparative studies. Surg Endosc 2012;26:904-13.

6. Dulucq JL, Wintringer P, Stabilini C, et al. Are major laparoscopic pancreatic resections worthwhile? A prospective study of 32 patients in a single institution. Surg Endosc 2005;19:1028-34.

7. Govil S, Imrie CW. Value of splenic preservation during distal pancreatectomy for chronic pancreatitis. Br J Surg 1999;86:895-8.

8. Tang CW, Feng WM, Bao Y, et al. Spleen-preserving distal pancreatectomy or distal pancreatectomy with splenectomy?: Perioperative and patient-reported outcome 
analysis. J Clin Gastroenterol 2014;48:e62-6.

9. Yan JF, Xu XW, Jin WW, et al. Laparoscopic spleenpreserving distal pancreatectomy for pancreatic neoplasms: a retrospective study. World J Gastroenterol 2014;20:13966-72.

10. Fernández-Cruz L, Orduna D, Cesar-Borges G, et al. Distal pancreatectomy: en-bloc splenectomy vs spleenpreserving pancreatectomy. HPB (Oxford) 2005;7:93-8.

11. Worhunsky DJ, Zak Y, Dua MM, et al. Laparoscopic spleen-preserving distal pancreatectomy: the technique must suit the lesion. J Gastrointest Surg 2014;18:1445-51.

12. Warshaw AL. Conservation of the spleen with distal pancreatectomy. Arch Surg 1988;123:550-3.

13. Yu X, Li H, Jin C, et al. Splenic vessel preservation versus Warshaw's technique during spleen-preserving distal pancreatectomy: a meta-analysis and systematic review. Langenbecks Arch Surg 2015;400:183-91.

14. Baldwin KM, Katz SC, Espat NJ, et al. Laparoscopic spleen-preserving distal pancreatectomy in elderly subjects: splenic vessel sacrifice may be associated with a higher rate of splenic infarction. HPB (Oxford) 2011;13:621-5.

15. Waters JA, Canal DF, Wiebke EA, et al. Robotic distal pancreatectomy: cost effective? Surgery 2010;148:814-23.

16. Hwang HK, Kang CM, Chung YE, et al. Robot-assisted spleen-preserving distal pancreatectomy: a single surgeon's experiences and proposal of clinical application. Surg Endosc 2013;27:774-81.

17. Niu X, Yu B, Yao L, et al. Comparison of surgical outcomes of robot-assisted laparoscopic distal pancreatectomy versus laparoscopic and open resections: A systematic review and meta-analysis. Asian J Surg 2019;42:32-45.

18. Gavriilidis P, Roberts KJ, Sutcliffe RP. Comparison of robotic vs laparoscopic vs open distal pancreatectomy. A systematic review and network meta-analysis. HPB (Oxford) 2019;21:1268-76.

19. Kang CM, Kim DH, Lee WJ, et al. Conventional laparoscopic and robot-assisted spleen-preserving pancreatectomy: does da Vinci have clinical advantages? Surg Endosc 2011;25:2004-9.

20. Benizri EI, Germain A, Ayav A, et al. Short-term perioperative outcomes after robot-assisted and laparoscopic distal pancreatectomy. J Robot Surg 2014;8:125-32.

21. Lee SY, Allen PJ, Sadot E, et al. Distal pancreatectomy: a single institution's experience in open, laparoscopic, and robotic approaches. J Am Coll Surg 2015;220:18-27.

22. Gavriilidis P, Lim C, Menahem B, et al. Robotic versus laparoscopic distal pancreatectomy - The first meta- analysis. HPB (Oxford) 2016;18:567-74.

23. Butturini G, Damoli I, Crepaz L, et al. A prospective nonrandomised single-center study comparing laparoscopic and robotic distal pancreatectomy. Surg Endosc 2015;29:3163-70.

24. Lee LS, Hwang HK, Kang CM, et al. Minimally Invasive Approach for Spleen-Preserving Distal Pancreatectomy: a Comparative Analysis of Postoperative Complication Between Splenic Vessel Conserving and Warshaw's Technique. J Gastrointest Surg 2016;20:1464-70.

25. Yongfei H, Javed AA, Burkhart R, et al. Geographical variation and trends in outcomes of laparoscopic spleenpreserving distal pancreatectomy with or without splenic vessel preservation: A meta-analysis. Int J Surg 2017;45:47-55.

26. Paiella S, De Pastena M, Korrel M, et al. Long term outcome after minimally invasive and open Warshaw and Kimura techniques for spleen-preserving distal pancreatectomy: International multicenter retrospective study. Eur J Surg Oncol 2019;45:1668-73.

27. Choi SH, Kang CM, Lee WJ, et al. Robot-assisted spleenpreserving laparoscopic distal pancreatectomy. Ann Surg Oncol 2011;18:3623.

28. Nakamura M, Ueda J, Kohno H, et al. Prolonged perifiring compression with a linear stapler prevents pancreatic fistula in laparoscopic distal pancreatectomy. Surg Endosc 2011;25:867-71.

29. Bassi C, Marchegiani G, Dervenis C, et al. The 2016 update of the International Study Group (ISGPS) definition and grading of postoperative pancreatic fistula: 11 Years After. Surgery 2017;161:584-91.

30. Lof S, Moekotte AL, Al-Sarireh B, et al. Multicentre observational cohort study of implementation and outcomes of laparoscopic distal pancreatectomy. Br J Surg 2019;106:1657-65.

31. van Hilst J, de Rooij T, Bosscha K, et al. Laparoscopic versus open pancreatoduodenectomy for pancreatic or periampullary tumours (LEOPARD-2): a multicentre, patient-blinded, randomised controlled phase 2/3 trial. Lancet Gastroenterol Hepatol 2019;4:199-207.

32. Røsok BI, de Rooij T, van Hilst J, et al. Minimally invasive distal pancreatectomy. HPB 2017;19:205-14.

33. Song KB, Kim SC, Park JB, et al. Single-center experience of laparoscopic left pancreatic resection in 359 consecutive patients: changing the surgical paradigm of left pancreatic resection. Surg Endosc 2011;25:3364-72.

34. Kang CM. Minimally Invasive (Laparoscopic or Robotic) Reduced Port (Single Port) Distal Pancreatectomy. J 
Minim Invasive Surg 2017;20:5-15.

35. Liu Y, Ji WB, Wang HG, et al. Robotic spleen-preserving laparoscopic distal pancreatectomy: a single-centered Chinese experience. World J Surg Oncol 2015;13:275.

36. Eckhardt S, Schicker C, Maurer E, et al. RoboticAssisted Approach Improves Vessel Preservation in Spleen-Preserving Distal Pancreatectomy. Dig Surg 2016;33:406-13.

37. Liu R, Liu Q, Zhao ZM, et al. Robotic versus laparoscopic distal pancreatectomy: A propensity score-matched study. J Surg Oncol 2017;116:461-9.

38. Mellemkjoer L, Olsen JH, Linet MS, et al. Cancer risk after splenectomy. Cancer 1995;75:577-83.

39. Sledzianowski JF, Duffas JP, Muscari F, et al. Risk factors for mortality and intra-abdominal morbidity after distal pancreatectomy. Surgery 2005;137:180-5.

40. Edgren G, Almqvist R, Hartman M, et al. Splenectomy and the risk of sepsis: a population-based cohort study. Ann Surg 2014;260:1081-7.

41. Barmparas G, Lamb AW, Lee D, et al. Postoperative infection risk after splenectomy: A prospective cohort study. Int J Surg 2015;17:10-4.

42. Yamaguchi K, Noshiro H, Yokohata K, et al. Is there any benefit of preservation of the spleen in distal pancreatectomy? Int Surg 2001;86:162-8.

43. Kimura W, Yano M, Sugawara S, et al. Spleen-preserving distal pancreatectomy with conservation of the splenic artery and vein: techniques and its significance. J Hepatobiliary Pancreat Sci 2010;17:813-23.

44. Warshaw AL. Distal pancreatectomy with preservation of the spleen. J Hepatobiliary Pancreat Sci 2010;17:808-12.

45. Ferrone CR, Konstantinidis IT, Sahani DV, et al. Twenty-three years of the Warshaw operation for distal pancreatectomy with preservation of the spleen. Ann Surg 2011;253:1136-9.

46. Jean-Philippe Adam, Alexandre J, Christophe L, et al.

Cite this article as: Yang SJ, Hwang HK, Kang CM, Lee WJ. Revisiting the potential advantage of robotic surgical system in spleen-preserving distal pancreatectomy over conventional laparoscopic approach. Ann Transl Med 2020;8(5):188. doi: 10.21037/atm.2020.01.80
Laparoscopic spleen-preserving distal pancreatectomy: splenic vessel preservation compared with the Warshaw technique. JAMA Surg 2013;148:246-52.

47. Lai EC, Tang CN. Robotic distal pancreatectomy versus conventional laparoscopic distal pancreatectomy: a comparative study for short-term outcomes. Front Med 2015;9:356-60.

48. Fuertes-Guiró F, Girabent-Farres M, Viteri-Velasco E. Opportunity cost in the economic evaluation of da Vinci robotic assisted surgery. Eur J Health Econ 2016;17:245-56.

49. Hua Y, Javed AA, Burkhart RA, et al. Preoperative risk factors for conversion and learning curve of minimally invasive distal pancreatectomy. Surgery 2017;162:1040-7.

50. Kim HS, Park JS, Yoon DS. True learning curve of laparoscopic spleen-preserving distal pancreatectomy with splenic vessel preservation. Surg Endosc 2019;33:88-93.

51. Nachmany I, Pencovich N, Ben-Yehuda A, et al. Laparoscopic Distal Pancreatectomy: Learning Curve and Experience in a Tertiary Center. J Laparoendosc Adv Surg Tech A 2016;26:470-4.

52. Shakir M, Boone BA, Polanco PM, et al. The learning curve for robotic distal pancreatectomy: an analysis of outcomes of the first 100 consecutive cases at a highvolume pancreatic centre. HPB (Oxford) 2015;17:580-6.

53. Shyr BU, Chen SC, Shyr YM, et al. Learning curves for robotic pancreatic surgery-from distal pancreatectomy to pancreaticoduodenectomy. Medicine (Baltimore) 2018;97:e13000.

54. Kim H, Kim JR, Han Y, et al. Early experience of laparoscopic and robotic hybrid pancreaticoduodenectomy. Int J Med Robot 2017. doi: 10.1002/rcs.1814.

55. Watkins AA, Kent TS, Gooding WE, et al. Multicenter outcomes of robotic reconstruction during the early learning curve for minimally-invasive pancreaticoduodenectomy. HPB (Oxford) 2018;20:155-65. 